\title{
Delocalization in coupled one-dimensional chains
}

\author{
P. W. Brouwer ${ }^{a}$, C. Mudry ${ }^{a}$, B. D. Simons ${ }^{a}$, and A. Altland ${ }^{b}$ \\ a Lyman Laboratory of Physics, Harvard University, MA 02138, USA \\ ${ }^{b}$ Institut für Theoretische Physik, Universität zu Köln, Zülpicher Strasse 77, 50937 Köln, Germany
}

(September 11, 2018)

\begin{abstract}
A weakly disordered quasi-one-dimensional tight-binding hopping model with $N$ rows is considered. The probability distribution of the Landauer conductance is calculated exactly in the middle of the band, $\varepsilon=0$, and it is shown that a delocalization transition at this energy takes place if and only if $N$ is odd. This even-odd effect is explained by level repulsion of the transmission eigenvalues.
\end{abstract}

PACS numbers: 72.15.Rn, 11.30.R

The existence of delocalization transitions in a disordered one-dimensional system is surprising, as it goes against the general wisdom that disordered systems in less than two dimensions are localized [1]. Nevertheless a delocalization transition in one dimension goes back to Dyson's work on models for a glass in 1953 [2,3. Dyson's one-dimensional glass is related to a large variety of disordered systems: a one-dimensional tight-binding model with nearest-neighbor random hopping [3], a twodimensional asymmetric random bond Ising model [4] which is equivalent to the one-dimensional random quantum Ising chain [5], one-dimensional random bond quantum XY models [6] and more generally random XYZ spin-1/2 Heisenberg models [7], and narrow gap semiconductors [8]. These models are of current interest in view of their rich physics: new universality classes, logarithmic scaling, the existence of strong fluctuations calling for a distinction between average and typical properties. They might also be useful laboratories to address the problem of disorder induced quantum phase transitions in higher dimensions such as the plateau transition between insulating Hall states in the quantum Hall effect [9, 10].

The one-dimensional nearest-neighbor random hopping model is described by the Hamiltonian

$$
\mathcal{H}=-\sum_{n}\left(t_{n} c_{n}^{\dagger} c_{n+1}+t_{n}^{*} c_{n+1}^{\dagger} c_{n}\right)
$$

where the operators $c_{n}^{\dagger}$ and $c_{n}$ are creation and annihilation operators for spinless fermions, respectively, and the hopping parameter $t_{n}=t+\delta t_{n}$ consists of a non-random part $t$ and a fluctuating part $\delta t_{n}$. The fundamental symmetry of the Hamiltonian (1) that distinguishes it from one-dimensional systems with on-site disorder is the presence of a sublattice (or chiral) symmetry: particles can hop only from even to odd-numbered sites. The energy $\varepsilon=0$ is special since it corresponds to a logarithmically diverging mean density of states [2]. Furthermore, there are several independent correlation lengths that diverge for $\varepsilon \rightarrow 0$ [7] indicating that the energy $\varepsilon=0$ represents a (disorder induced) quantum critical point [4, 6, 7, 9]. In particular, at $\varepsilon=0$ the conductance exhibits large fluctu- ations superimposed on an algebraically decaying mean value [10]. By contrast, for nonzero energy the system described by Eq. (11) is non-criticial resulting in standard localized behaviour: A typical sample is well characterized by $\langle\log g\rangle$, which is proportional to $L$ and has relatively small sample- to-sample fluctuations.

A different type of delocalization in one-dimensional disordered systems was considered recently by Hatano and Nelson [11], who considered a chain with on-site disorder and an imaginary vector potential. As a function of the strength of the imaginary vector potential, the system reaches a critical point and goes through a delocalization transition.

The discussion so far applies to the case of strictly onedimensional systems. In this Letter we address the question of whether aspects of the behaviour described above carry over to the multi-channel case. Surprisingly, it will turn out that the answer depends on the parity of the channel number $N$ : For $N$ even, the system behaves very much like standard disordered multi-channel wires, i.e. in the limit $L \rightarrow \infty$ all states are localized. However, for $N$ odd, precisely one mode remains critical and, moreover, exhibits much of the behaviour of the single critical mode of strictly one-dimensional systems. For large $L$, where the contribution of all other - localized - modes is neglegible, the phenomenology of the wire is determined by the contribution of the single critical mode, and, in this sense, remains critical. To our knowledge, this parity effect was first noticed by Miller and Wang in their study of random flux and passive advection models [12]. However, in that work, the effect has been washed out by taking a two-dimensional thermodynamic limit. Keeping $N$ finite we here focus on a different regime, where parity has pronounced phenomenological consequences.

To probe the onset of critical behaviour as $\varepsilon \rightarrow 0$ we calculate the probability distribution of the conductance. As will be shown below the even/odd effect manifests itself in the level repulsion between transmission eigenvalues. We also discuss the effect of staggering in the nonrandom part of the hopping parameter $t$ (connected e.g. to a Peierls instability) and establish a relation between delocalization transition in random hopping models and 
in non-Hermitian quantum mechanics.

To be specific, we consider the Hamiltonian

$$
\mathcal{H}=-\sum_{n} \sum_{i, j=1}^{N}\left(t_{n, i j} c_{n, j}^{\dagger} c_{n+1, i}+t_{n, i j}^{*} c_{n+1, i}^{\dagger} c_{n, j}\right) \text {, }
$$

where the indices $i$ and $j$ label the $N$ chains. Weak staggering in the hopping is introduced by setting $t_{n, i j}=$ $t \delta_{i j}+(-1)^{n} t^{\prime} \delta_{i j}+\delta t_{n, i j}$, where $t^{\prime} \ll t$. We distinguish between the cases in which time reversal symmetry is present $\left(\beta=1, t_{n, i j}\right.$ real) from those where it is absent ( $\beta=2, t_{n, i j}$ complex). The weakly fluctuating parts of the hopping amplitudes $\delta t_{n, i j}$ are taken to be independent and Gaussian distributed, with zero mean and with variance $\left\langle\delta t_{n, i j} \delta t_{n, i j}^{*}\right\rangle=\beta v^{2} / \gamma$, where $\gamma=\beta N+2-\beta$.

Upon linearization of the spectrum in the vicinity of the Fermi energy $\varepsilon=0$, the lattice model (2) can be approximated by a continuum model obeying the Schrödinger equation

$$
\begin{aligned}
\varepsilon \psi_{i}(y) & =\sum_{j=1}^{N} h_{i j}(y) \psi_{j}(y), \quad i=1, \ldots, N, \\
h_{i j} & =i v_{F} \delta_{i j} \sigma_{1} \partial_{y}+v_{i j}(y) \sigma_{1}+w_{i j}(y) \sigma_{2} .
\end{aligned}
$$

Here $\psi$ is a two-component wavefunction, corresponding to even and odd-numbered sites in the original lattice model, $y=2 n a, a$ being the lattice constant, and $v_{F}=2 t a$ is the Fermi velocity. The sublattice symmetry of the lattice model (2) translates to $\sigma_{3} h_{i j} \sigma_{3}=-h_{i j}$, which we refer to as chiral symmetry. The chiral symmetry distinguishes this system from one-dimensional systems with on-site disorder, which do not show a delocalization transition. The random potentials $v$ and $w$ are Hermitian $\left(v_{i j}=v_{j i}^{*}, w_{i j}=w_{j i}^{*}\right)$ while, in the presence of time-reversal symmetry, one has the further condition $v_{i j}=-v_{i j}^{*}$ and $w_{i j}=w_{i j}^{*}$. Apart from the symmetry constraints, the random potentials are independent and Gaussian distributed, with mean $\left\langle v_{i j}(y)\right\rangle=0$ and $\left\langle w_{i j}(y)\right\rangle=2 t^{\prime} \delta_{i j}$, and variance $\left(\bar{v}^{2}=2 v^{2} a \beta \gamma^{-1}\right)$

$$
\begin{aligned}
\left\langle\delta v_{i j}(y) \delta v_{i j}\left(y^{\prime}\right)^{*}\right\rangle & =\bar{v}^{2} \delta\left(y-y^{\prime}\right)\left(1-\delta_{\beta 1} \delta_{i j}\right) \\
\left\langle\delta w_{i j}(y) \delta w_{i j}\left(y^{\prime}\right)^{*}\right\rangle & =\bar{v}^{2} \delta\left(y-y^{\prime}\right)\left(1+\delta_{\beta 1} \delta_{i j}\right) .
\end{aligned}
$$

In order to find the conductance at zero energy, we calculate the distribution of the $2 N \times 2 N$ transfer matrix $M$, which relates wavefunctions at the left and right of a disordered strip of length $L \sqrt{13}$. The eigenvalues of $M M^{\dagger}$, which arise in inverse pairs $\exp \left( \pm 2 x_{j}\right)$, determine the transmission eigenvalues $T_{j}=1 / \cosh ^{2} x_{j}$ and hence the conductance $g$ through the Landauer formula

$$
g=\sum_{j=1}^{N} T_{j}=\sum_{j=1}^{N} \frac{1}{\cosh ^{2} x_{j}} .
$$

In the absence of disorder, all exponents $x_{j}$ are zero, and conduction is perfect, $g=N$. On the other hand, transmission is exponentially suppressed if all $x_{j}$ 's are larger than unity. The $x_{j}$ 's are related to the channel-dependent localization lengths $\xi_{j}=L /\left|x_{j}\right|$. The largest length $\xi$ determines the exponential decay of the conductance $g$ and serves as the localization length of the total system of coupled chains.

To compute the distribution of $M$, we use the FokkerPlanck approach pioneered for disordered wires with random on-site disorder by Dorokhov 14 and Mello, Pereyra, and Kumar 15. Following the method of Refs. [14,15, we first consider the case of disorder confined to a small strip $0<y<\delta L$. Denoting the wave function for $y<0$ by $\psi_{j}(L)$ and for $y>\delta L$ by $\psi_{j}(R)$, we find

$$
\psi_{j}(R)=\sum_{k=1}^{N} M_{j k} \psi_{k}(L)
$$

where the (random) transfer matrix $M$ of the slice reads

$$
M=T_{y} \exp \left\{v_{F}^{-1} \int_{0}^{\delta L} d y\left[i v(y)-\sigma_{3} w(y)\right]\right\}
$$

Here $T_{y}$ denotes the ordering operator for the $y$ integration.

For any given realization of the disorder, the transfer matrix has the following symmetry properties:

$$
\begin{aligned}
& \sigma_{3} M \sigma_{3}=M \text { (chiral symmetry), } \\
& M \sigma_{1} M^{\dagger}=\sigma_{1} \text { (flux conservation), } \\
& M^{*}=M \quad \text { (time reversal). }
\end{aligned}
$$

Taking the symmetries (7) into account, we find that the transfer matrix can be parameterized as

$$
M=u \exp \left(x \sigma_{3}\right) v
$$

where $u$ and $v$ are the tensor product of $N \times N$ unitary matrices (orthogonal if $\beta=1$ ) with the $2 \times 2$ unit matrix and $x$ is a diagonal $N \times N$ matrix with real diagonal elements $x_{1}, \ldots, x_{N}$. The numbers $x_{1}, \ldots, x_{N}$ are the radial coordinates of the transfer matrix (eigenvalues of $\frac{1}{2} \log M M^{\dagger}$ ), and the matrices $u$ and $v$ are the angular coordinates. In contrast to systems without chiral symmetry, the $x_{j}$ can be both positive and negative.

The transfer matrix of a system of length $L$ is found by multiplication of the transfer matrices of the many individual slices of width $\delta L$. As each multiplication results in a small change of the radial coordinates $x_{j}$, they perform a "Brownian motion" 14,15. Upon multiplication with the transfer matrix of a slice of width $\delta L$, we find that the radial coordinates $x_{j}$ change according to $x_{j} \rightarrow x_{j}+\delta x_{j}$, where the first two moments of the increment $\delta x_{j}$, averaged over the disorder configuration in the added slice, are given by 


$$
\begin{aligned}
\left\langle\delta x_{j}\right\rangle_{\delta L} & =\frac{\beta \delta L}{2 \ell \gamma}\left(-f+\sum_{k \neq j} \operatorname{coth}\left(x_{j}-x_{k}\right)\right), \\
\left\langle\delta x_{j} \delta x_{k}\right\rangle_{\delta L} & =\frac{\delta L}{\ell \gamma} \delta_{j k} .
\end{aligned}
$$

Here the mean free path $\ell$ and dimensionless staggeringdisorder ratio $f$ read

$$
\ell=v_{F}^{2} / 4 v^{2} a, \quad f=\gamma t^{\prime} v_{F} / v^{2} a \beta .
$$

The first term on the r.h.s. of Eq. (9a) results in a simultaneous drift of all radial coordinates $x_{j}$. The second term describes repulsion between nearby $x_{j}$ in the Brownian motion process. The Fokker-Planck equation corresponding to Eq. (9) reads

$$
\begin{aligned}
\ell \frac{\partial P}{\partial L} & =\frac{1}{2 \gamma} \sum_{j=1}^{N} \frac{\partial}{\partial x_{j}}\left(\beta f+J \frac{\partial}{\partial x_{j}} J^{-1}\right) P, \\
J & =\prod_{k>j}\left|\sinh \left(x_{j}-x_{k}\right)\right|^{\beta} .
\end{aligned}
$$

The initial condition corresponding to perfect transmission at $L=0$ is $P\left(x_{1}, \ldots, x_{N} ; 0\right)=\prod_{j} \delta\left(x_{j}\right)$.

The Fokker-Planck equation (10) is the central result of this Letter [16]. It contains all information on the transport properties of the random hopping system at zero energy. Eq. (10) is the chiral analogue of the socalled Dorokhov-Mello-Pereyra-Kumar (DMPK) equation, which governs the evolution of the transmission eigenvalues of a disordered wire 13 15. The key difference between the two equations is the presence of "mirror imaged" eigenvalues $x_{j}$ in the DMPK equation, which are absent in Eq. (10). [For wires with on-site disorder, the eigenvalues $x_{j}$ not only repel from different eigenvalues $x_{k}$, c.f. Eq. (9a), but also from the "mirror image" $-x_{k}$; in particular, $x_{j}$ and $-x_{j}$ repel.]

In the absence of time-reversal symmetry $(\beta=2)$, the DMPK equation has been solved exactly by Beenakker and Rejaei 17] by a mapping to a problem of noninteracting fermions. Using the method of Ref. [17, we have been able to find an exact solution of Eq. (10) for $\beta=2$. It reads

$$
\begin{aligned}
P= & c(L) \prod_{j} \exp \left(-f x_{j}-x_{j}^{2} \frac{N \ell}{L}\right) \\
& \times \prod_{k>j}\left(x_{k}-x_{j}\right) \sinh \left(x_{k}-x_{j}\right),
\end{aligned}
$$

where $c(L)$ is a normalization constant. The exact solution (10) has a formal analogy to the distribution of eigenvalues of a random matrix: it consists of a pair interaction and a potential part. However, while for random matrices the eigenvalue interaction is quadratic, here we find a more complicated level repulsion. Comparing our result (11) to the exact solution of Beenakker and Rejaei, we note the absence of the mirror-image eigenvalues in the interaction and potential factors. No exact solution of Eq. (10) for $\beta=1$ could be found.

To determine whether the system is at a critical point, we investigate the distribution of $x_{j}$ 's for $L \rightarrow \infty$, which can be obtained from Eq. (9) for both $\beta=1$ and $\beta=2$. For $L \gg N \ell$, the radial coordinates $x_{j}$ are well separated, say $x_{1} \ll \ldots \ll x_{N}$. We then find from Eq. (9) that the "dynamics" of the $x_{j}$ 's $(j=1, \ldots, N)$ separate, and that they show small Gaussian fluctuations around equidistant equilibrium positions,

$$
\left\langle x_{j}\right\rangle=(N+1-2 j-f) L \beta / 2 \ell \gamma, \quad \operatorname{var} x_{j}=L / \gamma \ell .
$$

This is the so-called "crystallization of transmission eigenvalues" 13], which is a signature of localization in wires with on-site disorder. Transmission is exponentially suppressed if all radial coordinates $x_{j}$ are larger than unity, c.f. Eq. (बi). For on-site disorder all $x_{j}$ 's grow linearly with $L$ [13], which inevitably leads to strong localization. [Within the framework of the DMPK equation, this results from the repulsion between $x_{j}$ and the mirror image $-x_{j}$.] The situation is different for the coupled random hopping chains, where we find from Eq. (12) that the radial coordinate $x_{j}$ remains (on average) close to zero, thus resulting in a delocalized state and a critical point, provided

$$
N+1-2 j-f=0 .
$$

As a result, in the absence of staggering $(f=0)$, a critical point exists only if the number of chains is odd. If there is no staggering, an even number of coupled random hopping chains show an exponential decay of the conductance. The conductance distribution at the critical point follows directly from the Landauer formula (4) and the Gaussian distribution of the radial coordinate $x_{j}$. As fluctuations of $x_{j}$ around zero are large [see Eq. (12)], the conductance at the critical point shows large sample-to-sample fluctuations, and the random hopping chains at the critical point can by no means be regarded as a "good conductor".

The parity effect for the presence of a critical point in the absence of staggering can be understood from the "level repulsion" of the variables $x_{j}$. In the large- $L$ limit, where $x_{1} \ll \ldots \ll x_{N}$, the coordinates $x_{j}$ repel by constant forces, see Eq. (9a). For an even number of channels, there is a net force on all $x_{j}$ 's, driving them away from 0 and resulting in an exponential suppression of the conductance (see Fig. 17a). However, as is depicted in Fig. 1 $\mathrm{b}$, if the number of channels is odd, there is no force on the middle exponent $x_{(N+1) / 2}$. Therefore, this variable will remain close to zero and give rise to a diverging localization length $\xi=L /\left|x_{(N+1) / 2}\right|$ and a critical state. For comparison, in the case of a wire with on-site disorder, the repulsion between $x_{j}$ and its mirror image $-x_{j}$ 
results in a nonvanishing force for all radial coordinates [13] (see Fig. 11c).

By fine tuning the staggering parameter $f(9 \mathrm{~d})$, which measures the ratio of the uniform staggering $t^{\prime}$ and the random disorder strength $v$, an additional $[N / 2]$ critical points can be reached, both for even and odd number of chains $([N / 2]$ is the largest integer $\leq N / 2)$. According to Eq. (12), as the staggering parameter $f$ approaches the critical value $f=2 j-N-1$, the localization length $\xi=\xi_{j}=L /\left|x_{j}\right|$ diverges with (critical) exponent 1 .

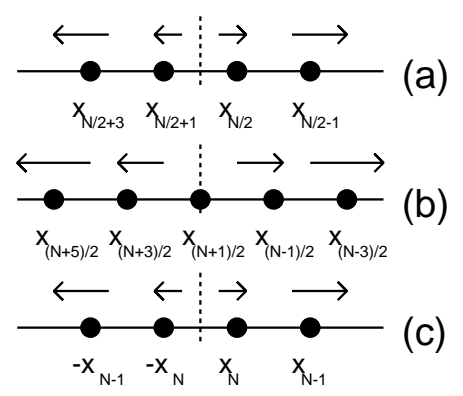

FIG. 1. The parity effect results from level repulsion between the transmission eigenvalues. (a) For an even number of chains, all radial coordinates $x_{j}$ are repelled away from 0 , while (b) for an odd number of chains $x_{(N+1) / 2}$ remains close to 0 . (c) Repulsion from mirror images for a wire with on-site disorder results in a positive driving force for all $x_{j}$.

The fact that we find exponential suppression of the conductance if Eq. (13) is not obeyed, may be due to either the existence of localized states, or to a gap in the spectrum. For instance, in Peierls materials, staggering opens a gap in the excitation spectrum, which explains the exponential suppression of the transmission even for zero disorder. The presence of disorder leads to a finite density of states below the excitation gap, but these subgap states are localized except for critical realizations of the disorder strength that satisfy Eq. (13).

To close, we discuss the relation between the critical points for the multi-chain random hopping model studied in this Letter and the Hatano-Nelson delocalization transition in (one-dimensional) non-Hermitian quantum mechanics [11]. The relation between the two systems is established through the "method of Hermitization" [18], in which the non-Hermitian problem with "Hamiltonian" $h$ at (complex) energy $z$ is made Hermitian by considering the Hamiltonian $H_{z}=\sigma_{1} \operatorname{Re}(h-z)+\sigma_{2} \operatorname{Im}(h-z)$. An eigenfunction of $h$ at eigenvalue $z$ is an eigenfunction of $H_{z}$ at eigenvalue 0 and vice versa. For complex disorder in the the non-Hermitian system, we find that the $N$-chain non-Hermitian problem maps to $2 N$ coupled chains with Hermitian quantum mechanics and with chiral symmetry. The staggering parameter in the chiral system plays the role of an imaginary vector potential considered by Hatano and Nelson 11. Thus, comparing the non-hermitian problem with the random hopping chain containing an even number of rows, we deduce that, in the absence of the imaginary vector potential, the non-Hermitian system is localized. Since the imaginary vector potential maps to the staggering, a series of $N$ critical points (and the corresponding branches of delocalized states with complex energy, see Ref. [11]) can then be obtained by tuning the values of the imaginary vector potential.

We are indebted to D. S. Fisher and B. I. Halperin for useful discussions. One of us (AA) would like particularly to acknowledge important discussions with J. T. Chalker at an early stage of this project, concerning both the physical background of the problem and its formulation. PWB acknowledges support by the NSF under grants no. DMR 94-16910, DMR 96-30064, and DMR 94-17047. CM acknowledges a fellowship from the Swiss Nationalfonds.

* Permanent address: Cavendish Laboratory, Madingley Road, Cambridge, CB3 0HE (UK).

[1] N. F. Mott and W. D. Twose, Adv. Phys. 10, 107 (1961); R. E. Borland, Proc. R. Soc. Lond. A 274, 529 (1963).

[2] F. J. Dyson, Phys. Rev. 92, 1331 (1953).

[3] G. Theodorou and M. H. Cohen, Phys. Rev. B 13, 4597 (1976); T. P. Eggarter and R. Riedinger, Phys. Rev. B 18, 569 (1978).

[4] B. M. McCoy and T. T. Wu, Phys. Rev. 176, 631 (1968).

[5] R. Shankar and G. Murphy, Phys. Rev. B 36, 536 (1987).

[6] E. R. Smith, J. Phys. C 3, 1419 (1970); R. H. McKenzie, Phys. Rev. Lett. 77, 4804 (1996).

[7] D. S. Fisher. Phys. Rev. B 50, 3799 (1994); ibid 51, 6411 (1995).

[8] L. V. Keldysh, Zh. Eksp. Theor. Fiz. 45, 364 (1963) [Sov. Phys. JETP 18, 253 (1964)]; A. A. Ovchinnikov and N. S. Erikhman, Zh. Eksp. Teor. Fiz. 73, 650 (1977) [Sov. Phys. JETP 46, 340 (1977).]

[9] L. Balents and M. P. A. Fisher, Phys. Rev. B 56, 12970 (1997).

[10] H. Mathur, Phys. Rev. B 56, 15794 (1997).

[11] N. Hatano and D. R. Nelson, Phys. Rev. Lett. 77, 570 (1996).

[12] J. Miller and J. Wang, Phys. Rev. Lett. 76, 1461 (1996).

[13] For reviews, see: A. D. Stone, P. A. Mello, K. A. Muttalib, and J.-L. Pichard in Mesoscopic Phenomena in Solids, edited by B. L. Altshuler, P. A. Lee, and R. A. Webb (North Holland, Amsterdam, 1991); C. W. J. Beenakker, Rev. Mod. Phys. 69, 731 (1997).

[14] O. N. Dorokhov, Pis'ma Zh. Eksp. Teor. Fiz. 36, 259 (1982) [JETP Letters 36, 318].

[15] P. A. Mello, P. Pereyra, and N. Kumar, Ann. Phys. (NY) 181, 290 (1988).

[16] An analogous treatment of chiral quasi one-dimensional transport problems has independently been suggested by J. T. Chalker.

[17] C. W. J. Beenakker and B. Rejaei, Phys. Rev. Lett. 71, 3689 (1993); Phys. Rev. B 49, 7499 (1994).

[18] H. J. Sommers et al., Phys. Rev. Lett. 60, 1895 (1988); J. Feinberg and A. Zee, Nucl. Phys. B504 [FS], 579 (1997). 